\title{
Exemple de prévision et de simulation opérationnelle des débits d'étiage pour les besoins d'EDF
}

\author{
An example of forecasting and operating simulation of low water flows
}

\author{
par R. Garçon, C. Carré, P. Lyaudet
}

EDF-Division Technique Générale

In low water level situations, it is hard to manage all water resource requirements. EDF, in particular, uses water for energy purposes both for heat production means which require a minimum flow for their cooling and for dilution of their effluents, and for its hydraulic production means whose reserves allow expensive fuels to be saved during peak demand periods. Use of water resources must also take into account the additional demand corresponding to other needs such as agricultural, industrial, tourist or related to water quality. In such conditions, to ensure optimum management of a dam, knowledge and short-term forecasting of natural flows enable water to be released in sufficient quantity to satisfy downstream needs while ensuring maximum water savings to cope with future needs. Moreover, simulation of long-term hydrological scenarios makes it possible to forecast the occurrence of events identified as critical, such as the overshooting of certain flow thresholds or their persistence in time, in order to anticipate the consequences of these events on operation of the installed production base. EDF is therefore gradually acquiring the tools needed for knowledge and forecasting of water resources in low water level situations: pliviometric and hydrometric stations, conceptual and statistical hydrological forecast models, data servers, low water level indicator statistical analysis models, water resource indicators. This approach is illustrated by the current achievements and developments in the Vienne and Loire basins.

\section{I $\square$ LA PROBLÉMATIQUE DES ÉTIAGES À EDF}

\subsection{L'eau et la production d'électricité}

EDF en tant que gestionnaire d'aménagements hydroélectriques, dispose de réserves d'eau conséquentes. On dénombre parmi l'ensemble des réserves environ 80 dont l'intérêt énergétique est marqué et dont la capacité utile avoisine les $6000 \mathrm{hm}^{3}$.

Cette eau est d'abord dédiée à la production d'énergie électrique. On rappelle que l'énergie hydroélectrique représente $15 \%$ de la production totale à EDF. Ce type d'énergie. comme chacun sait, permet l'économie de combustibles onéreux lors des pointes de consommation journalière.

Cependant, en période d'étiage, d'autres moyens de production d'électricité peuvent faire appel à ces réserves. Ainsi, le parc nucléaire dans un contexte d'étiage marqué a besoin d'apports en eau supplémentaires. En effet, le fonctionnement d'une centrale nucléaire située au droit d'une rivière, nécessite un débit minimal d'écoulement. Ce débit, fixé par arrêté, permet entre autres le maintien à la normale de la température de l'eau de la rivière quand celle-ci est uti- lisée comme source froide au sein de la centrale. De plus, des conventions fixent un débit minimal de rejet des effluents radio-actifs en milieu naturel. Ainsi, en période de sécheresse, la capacité des cuves de stockage de ces effluents peut arriver à saturation. Des apports supplémentaires sont alors nécessaires pour diluer les effluents et permettre à la centrale de continuer à fonctionner.

Le besoin en eau en période de sécheresse se fait logiquement plus particulièrement ressentir pour les centrales nucléaires à proximité de rivières de moyenne envergure, ainsi la Vienne à Civaux ou la Meuse à Chooz. Cependant, on constate aussi un besoin de surveillance accrue sur des rivières dont le débit est plus important telle la Garonne à Golfech.

\subsection{Les autres usages de l'eau}

Bien que disposant des réserves les plus importantes, EDF n'est pas le seul " acteur de l'eau ". En période d'étiage, d'autres préoccupations peuvent apparaître, externes à EDF.

Ainsi, dans le domaine de la qualité de l'eau, tant au niveau de la potabilité que de la survie piscicole, des apports supplémentaires peuvent éviter une dégradation du milieu. 
Par exemple, sur la Vienne, une convention permettait l'utilisation des réserves amont pour maintenir la qualité de l'eau dégradée par les rejets des industries. Il en est de même sur la Garonne ou le Lot.

On peut aussi citer les besoins en eau pour l'irrigation. Bien que n'ayant pas d'aménagement sur le bassin de la Charente, EDF a élaboré un tableau de bord de la ressource en eau en Charente pour aider à la gestion des réserves disponibles face à la demande des agriculteurs.

Enfin, l'activité touristique génère des besoins, tant au niveau des plans d'eau ou autres bases nautiques dont la cote doit être maintenue, qu'en termes de débit pour la pratique de sports d'eau vive.

\subsection{La gestion de la ressource en eau}

En parallèle à l'exploitation énergétique de ses réserves hydrauliques, EDF doit prendre en compte l'ensemble des besoins liés au manque d'eau en période d'étiage marqué : tant en interne pour le fonctionnement de certaines centrales nucléaires que vis-à-vis des collectivités locales, industriels ou agriculteurs...

La gestion des réserves doit être rigoureuse en tenant compte des différents besoins et de l'évolution à venir de l'étiage en cours. Ainsi, EDF se dote progressivement d'outils d'aide à la décision. Ils permettent une meilleure connaissance de l'état de la ressource en eau, et apportent une anticipation à plus ou moins long terme de son évolution.

On illustre cette démarche par deux exemples en cours de développement, l'un concerne la Centrale de Civaux sur la Vienne, l'autre l'ensemble des centrales nucléaires de la Loire.

\section{II $\square$ L'EXEMPLE DE LA VIENNE AU DROIT DE LA CENTRALE DE CIVAUX}

\subsection{Problématique}

La problématique de la Vienne au droit de la centrale de Civaux est la suivante. Le fonctionnement de la centrale de Civaux nécessite pour respecter les conventions liant EDF (concessionnaire de l'aménagement) et l'Etat, un débit minimal en rivière de l'ordre de $10 \mathrm{~m}^{3} / \mathrm{s}$.

En amont, EDF dispose de plusieurs réserves sur les chaînes Maulde et Taurion dont la réserve de Vassivière (voir la figure 1). Mais, la gestion de ces retenues n'influence le débit de la Vienne à Civaux qu'au terme d'un délai de propagation supérieur à 24 heures en basses eaux.

D'autre part, immédiatement en amont de la centrale, les usines du groupement de La Roche gérées au fil de l'eau disposent d'une réserve " tampon" de moins d' $1 \mathrm{hm}^{3}$.

L'objectif de la gestion de ce bassin est de maintenir le débit nécessaire au fonctionnement de la centrale à l'aide des réserves amont tout en maintenant l'activité hydroélectrique des chaînes amont et en tenant compte de la contrainte de cote sur la réserve de Vassivière en période touristique.

\subsection{Stratégie}

La stratégie adoptée consiste à anticiper à court terme (quelques jours) les apports naturels de la Vienne de façon à compléter ces apports par des lâchures en amont pour assurer le maintien du débit minimal en rivière. Cette gestion doit prendre en compte le délai de propagation des lâchures.

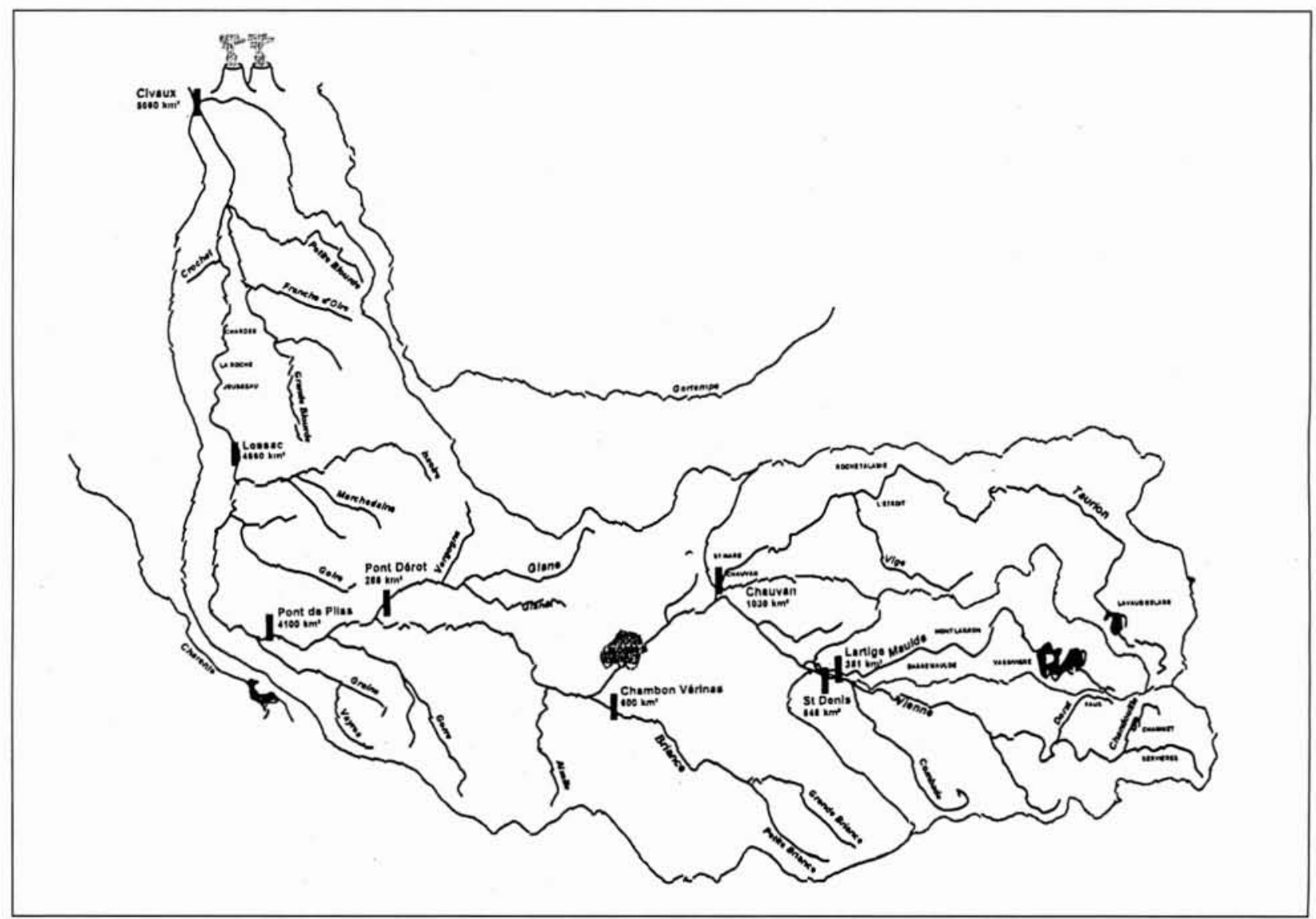

1. Carte et schématisation du bassin versant de la Vienne à Civaux. 
Les réserves du groupement de La Roche peuvent par ailleurs réguler le débit immédiatement en amont de la centrale à hauteur de sa capacité. Ces réserves doivent donc être constamment alimentées pour parer à toute éventualité.

Cette stratégie nécessite l'anticipation à court terme des apports naturels de la Vienne.

\subsection{Schématisation du bassin}

Pour permettre cette anticipation, le bassin de la Vienne a été schématisé de la façon suivante (voir figure 1). On distingue :

- le bassin versant naturel de la Vienne amont,

- les chaînes hydroélectriques de la Maulde et du Taurion,

- le bassin versant intermédiaire de la Vienne jusqu'à Lessac,

- le groupement de La Roche.

L'aide à la gestion de la ressource en eau comprend deux temps. Tout d'abord, la prévision à court terme des apports naturels de la Vienne amont et du bassin versant intermédiaire. Puis, la schématisation et l'optimisation des lâchures des chaînes amont en fonction du débit minimal nécessaire et du remplissage des réserves du groupement de La Roche.

\subsection{Prévision de débit à court terme (pas journalier)}

Le modèle hydrologique MORDOR simule au pas journalier le fonctionnement hydrologique d'un bassin versant. II est articulé autour de 4 réservoirs représentant les différentes couches du sol.

Le réservoir de surface correspondant à la couche superficielle du sol est alimenté par la pluie et soumis au phénomène d'évaporation de sol, fonction de la température externe. Un réservoir intermédiaire permet le contrôle de l'écoulement. Selon la pluviométrie, la contribution de ce réservoir peut être assimilée à un écoulement hypodermique ou à un ruissellement superficiel résultant d'un engorgement du sol. Un réservoir évaporant correspond à la couche radiculaire de la végétation sur le sol. Il simule l'influence de cette végétation sur l'écoulement par le phénomène d'évapotranspiration. Enfin, le dernier réservoir est assimilé à la couche profonde du sol ou nappe. Sa contribution est permanente, elle est de type " débit de base ".
A partir de ces quatre réservoirs, le modèle appréhende de façon schématique le parcours de la pluie depuis son impact sur le sol jusqu'à l'exutoire, cheminement traduit en termes de flux entre réservoirs ou vers l'extérieur. La figure 2 donne un exemple de simulation du modèle MORDOR sur une année complète.

L'application de ce modèle sur un bassin versant, moyennant l'anticipation des paramètres météorologiques que sont la précipitation et la température (appelés entrants du modèle), permet l'anticipation à quelques jours de l'évolution naturelle du débit.

Cependant, dans un contexte opérationnel, cette modélisation a été complétée par des développements supplémentaires que l'on a appelés " recalage opérationnel ". En effet, malgré le soin apporté lors de l'élaboration du modèle, de sa calibration et de la mesure des précipitations, on est condamné, compte tenu de l'état de l'art en hydrologie, à observer de temps à autre des écarts significatifs entre débits mesurés et simulés.

Ainsi, pour assurer la cohérence des prévisions avec la chronologie des débits observés, il est nécessaire de comparer le comportement des deux chronologies sur le passé. Une analyse statistique des écarts permet d'appliquer en temps réel une correction à la prévision initiale (issue de la simulation brute) pour élaborer une prévision correcte.

\subsection{Application au bassin de la Vienne}

Le modèle hydrologique MORDOR a été intégré dans la gestion de la ressource en eau de la Vienne. Il permet d'anticiper les débits naturels de la Vienne amont.

D'autre part, pour appréhender les apports naturels du bassin versant intermédiaire, le même modèle a été appliqué sur deux des affluents de la Vienne, la Briance et la Glane, permettant l'anticipation de leur évolution à court terme. La combinaison de ces résultats permet d'anticiper l'évolution d'un index naturel du bassin versant intermédiaire. Une dernière étape prenant en compte la composante volumique du bassin versant intermédiaire sur les valeurs observées permet d'élaborer les prévisions d'apports naturels du bassin versant intermédiaire.

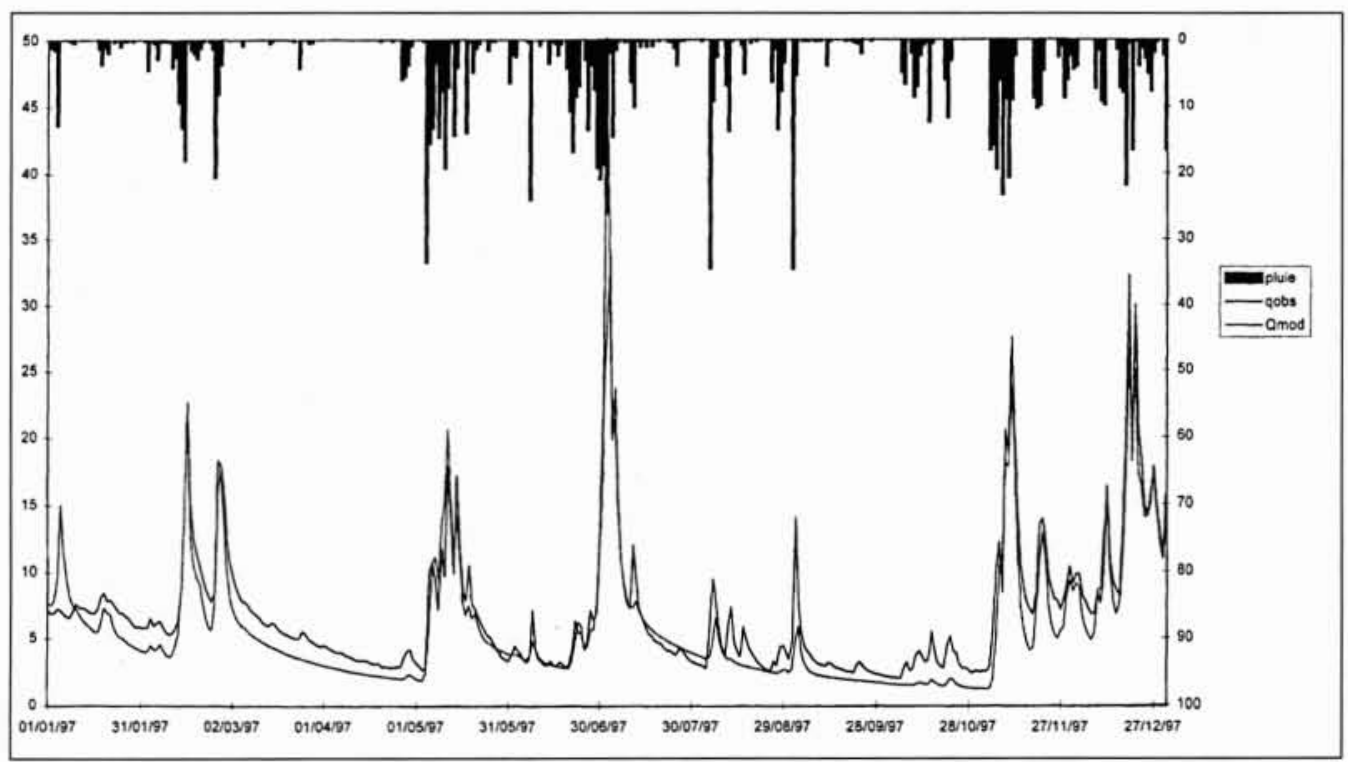

2. Débits observés et simulés sur le bassin de la Briance à Chambon Vérinas (année 97). 


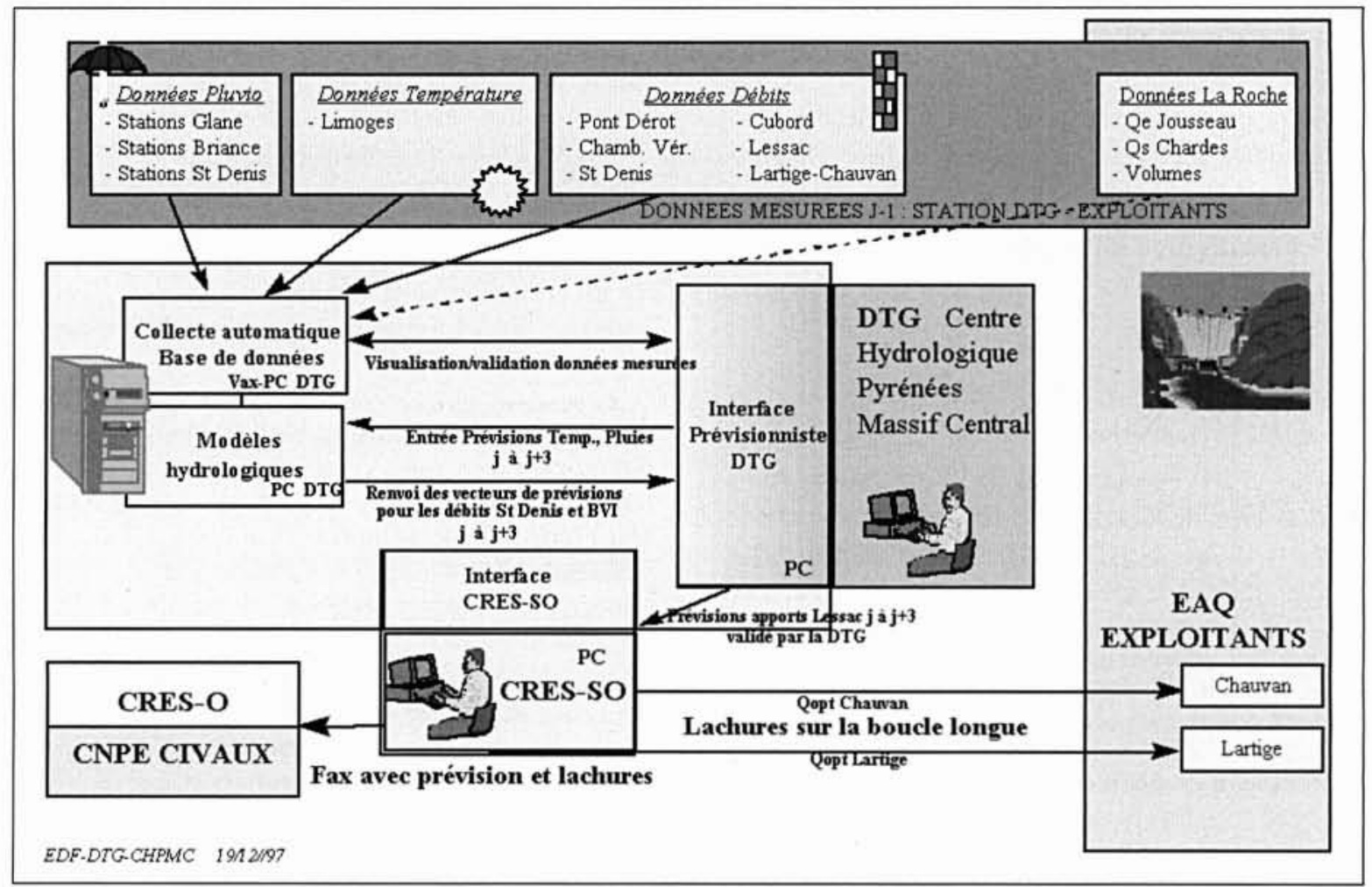

3. Système Opérationnel Vienne d'Aide à la Gestion des Etiages.

On dispose ainsi des prévisions de l'ensemble des apports naturels de la Vienne en amont de Civaux.

\subsection{Système Opérationnel Vienne d'Aide à la Ges- tion des Etiages (SOVAGE)}

Disposant de l'ensemble de ces développements, EDF a élaboré un outil d'aide à la gestion de la ressource.

Cet outil permet de visualiser tant d'un point de vue chronologique que spatial l'état hydrologique du bassin. Pour cela, on s'appuie sur un réseau de mesures (pluie-débit-température) transmises en temps réel.

D'autre part, les différents développements décrits ci-dessus (MORDOR, recalage opérationnel, index naturel...) ont été intégrés permettant une anticipation à court terme de l'évolution des apports naturels.

Enfin, l'outil contient un module de simulation des lachures des chaînes Maulde et Taurion dans un contexte optimal par rapport au soutien d'étiage (voir figure 3 ).

Cet outil permet à EDF de visualiser l'état de la ressource en eau et d'apporter des éléments de décision quant à sa gestion par anticipation à court terme.

\section{III - EXEMPLE DU BASSIN DE LA LOIRE AVAL}

\subsection{Problématique}

L'enjeu sur le bassin de la Loire aval avec plus de quatre centrales nucléaires en fonctionnement est important en période de sécheresse. La problématique est cependant différente de celle du bassin de la Vienne présentée précédemment.
En effet, contrairement à une anticipation à court terme pour le maintien d'un débit minimal de fonctionnement en rivière sur la Vienne, sur la Loire, c'est le moyen-long terme qui prédomine. La problématique se pose plus en termes de gestion des stocks d'effluents qu'en termes de débit minimal de fonctionnement. Si le débit est trop faible pendant une période importante, les centrales nucléaires doivent stocker leurs effluents dans la limite des capacités disponibles. Aussi, une anticipation à moyen long terme permet-elle de gérer au mieux ces stocks et de prévoir d'éventuels apports supplémentaires.

\subsection{La courbe enveloppe de tarissement}

Au début de la période d'étiage, aux environs de la mijuin, EDF a besoin d'appréhender le caractère critique ou non de l'étiage à venir en fonction de l'état hydrologique du bassin (il est clair que l'on ne peut pas avoir d'anticipation sur les précipitations de l'été à venir).

Pour cela, un modèle a été développé par D.DUBAND en 1972. Il permet d'estimer un faisceau de courbes enveloppes de tarissement graduées en probabilité suivant les conditions météorologiques de l'été, et dont la position dépend de l'état initial de saturation du bassin.

On définit la courbe enveloppe de tarissement comme l'enveloppe inférieure d'un hydrogramme en période d'étiage. En d'autres termes c'est la courbe qui tangente l'hydrogramme et enveloppe les points bas. Ce n'est pas une simulation des débits, mais le niveau de tarissement probable sur la période d'étiage.

L'analyse statistique sur laquelle repose ce modèle a montré la pertinence d'un calcul à la mi-juin. En effet, sur 110 ans de données (1863-1972), la répartition des dates 


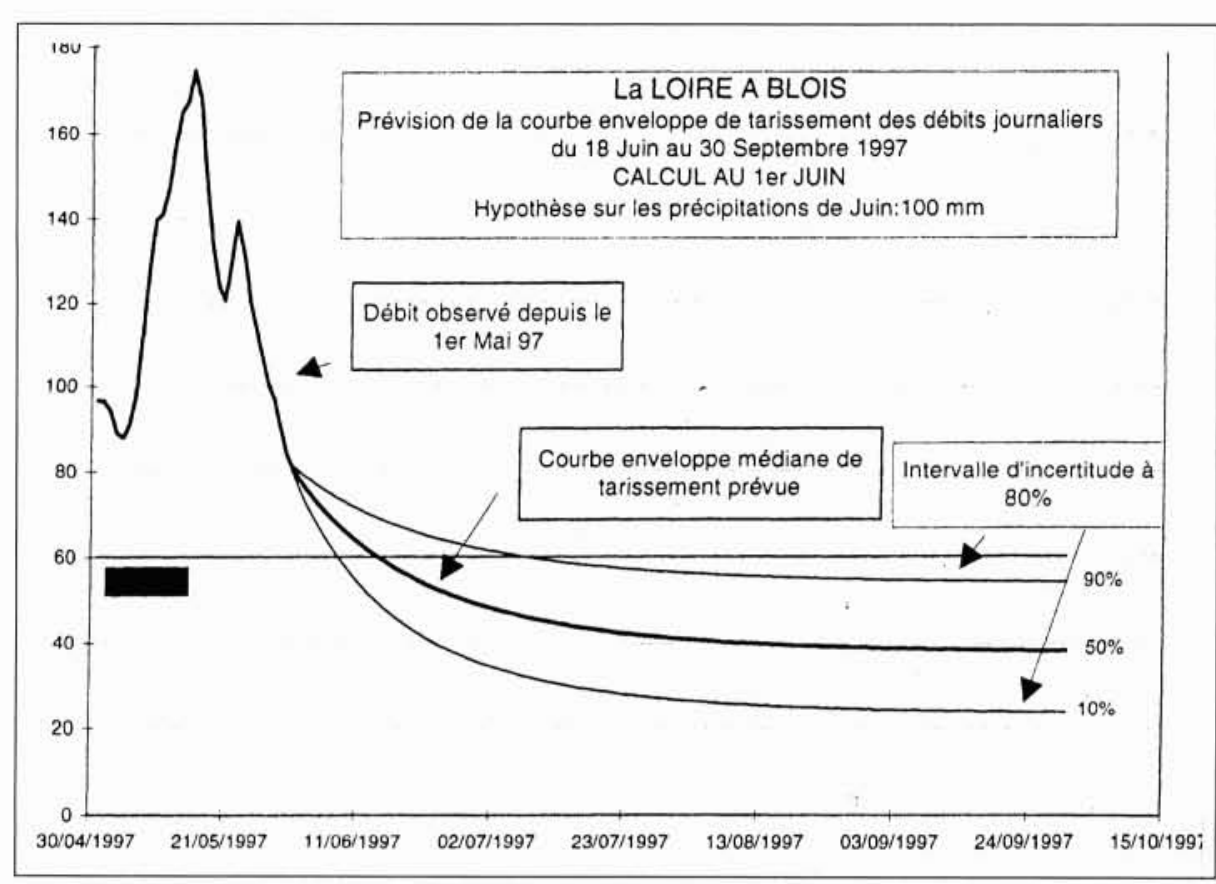

4. Exemple de prévision de courbe enveloppe de tarissement.

mer une évolution probable des débits pour l'année en cours à partir d'une échéance donnée graduée en terme de probabilité. On rappelle qu'on ne peut anticiper qu'une évolution naturelle du débit (sans considérer un éventuel soutien d'étiage).

L'intérêt de ces calculs réside dans la prise en compte de l'aléa météorologique sur les deux mois d'été. Ainsi, on a pu observer que, pour un passé semblable, les débits de la période estivale pouvaient retrouver des niveaux élevés (exemple de l'année 1875) grâce à des précipitations importantes ou tomber à des valeurs très faibles (exemple de l'année 1948). Cette variabilité apparaît bien évidemment dans l'analyse statistique sous forme de quantiles (voir figure 5) et complète avec

d'étiages minimum donne des chances d'occurrence appréciables à partir de la fin juin. Cela souligne l'importance des précipitations du mois de juin sur la période d'étiage qui suit : une crue importante peut " décaler " notablement le tarissement des débits sur la période d'été.

D'un point de vue opérationnel, ce modèle est mis en œuvre à la mi-juin avec une hypothèse sur les précipitations du mois de juin, puis réactualisé début juillet avec les valeurs de précipitations observées (voir exemple de prévision figure 4).

\section{- 3.3 Analyse statistique}

Devant la situation de sécheresse marquée au printemps dernier (1997), des développements supplémentaires ont été menés pour préciser les résultats de la courbe enveloppe " classiquement " proposée. Ils ont permis de moduler l'évolution possible de l'étiage et d'apporter des éléments précis d'aide à la décision pour la gestion de l'ensemble des réserves amont disponibles.

Ainsi, une vingtaine d'années analogues à l'année en cours en termes d'hydraulicité et de pluviométrie sur les mois de mars à mai ont été sélectionnées. La connaissance d'une part des débits observés lors de l'étiage qui a suivi, et d'autre part de la simulation de la courbe enveloppe de tarissement, a permis de moduler les résultats de la courbe enveloppe et de les traduire en termes de risque.

Dans un premier temps, en considérant l'évolution réelle des débits observés sur les années analogues, on peut esti- intérêt les résultats bruts de la courbe enveloppe.

Soulignons l'intérêt de cette analyse statistique s'appuyant sur une modélisation de la courbe enveloppe. Elle permet d'aller au-delà de la simple analyse de la distribution libre puisqu'elle est conditionnée par le passé de l'année en cours.

D'autre part, à partir de l'évolution probabilisée des débits, on peut tirer d’autres renseignements. Il s'est avéré intéressant d'extraire de ces résultats un risque en termes de débit minimal d'étiage ou de nombre de jours sous un seuil de débit fixé. Enfin, par rapport à l'aide à la gestion, une traduction en termes de volume de soutien nécessaire par rapport à un débit objectif permet de bien préciser la situation en regard des volumes réellement disponibles.

Au cours de l'été 97, quatre échéances ont été traitées. On peut apprécier par retour d'expérience la pertinence des prévisions proposées malgré le soutien d’étiage non pris en

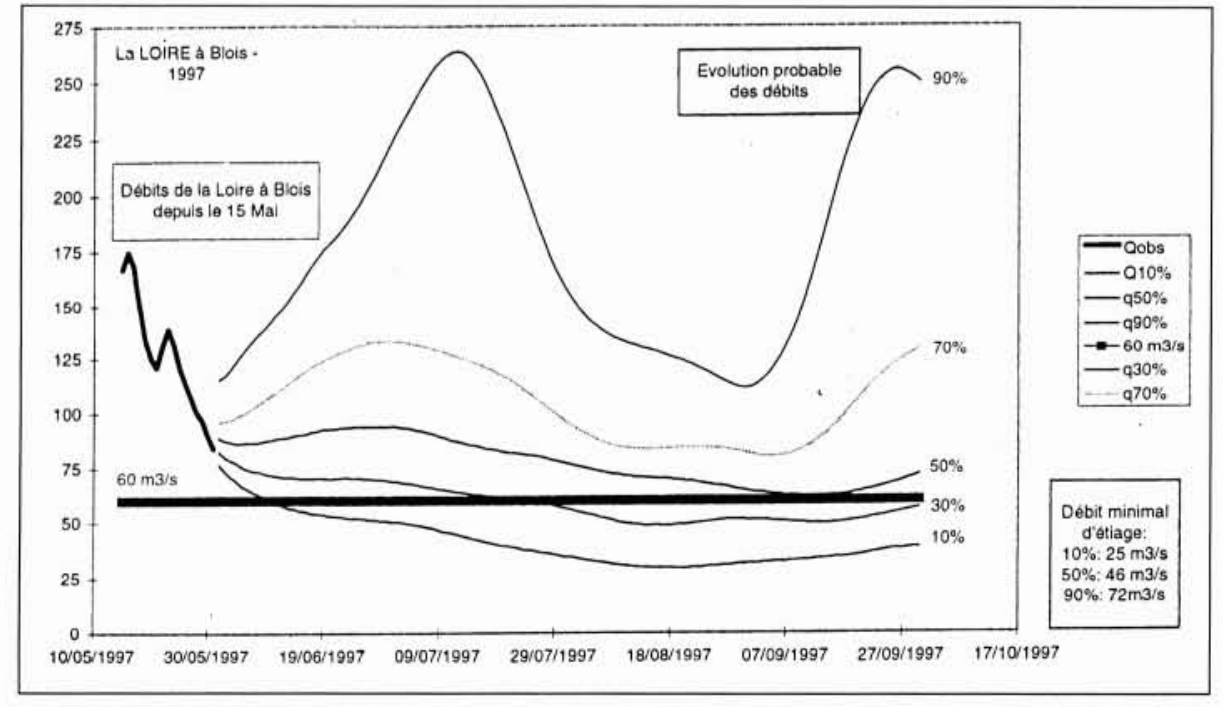

5. Exemple de prévision après traitement statistique de la courbe enveloppe. 


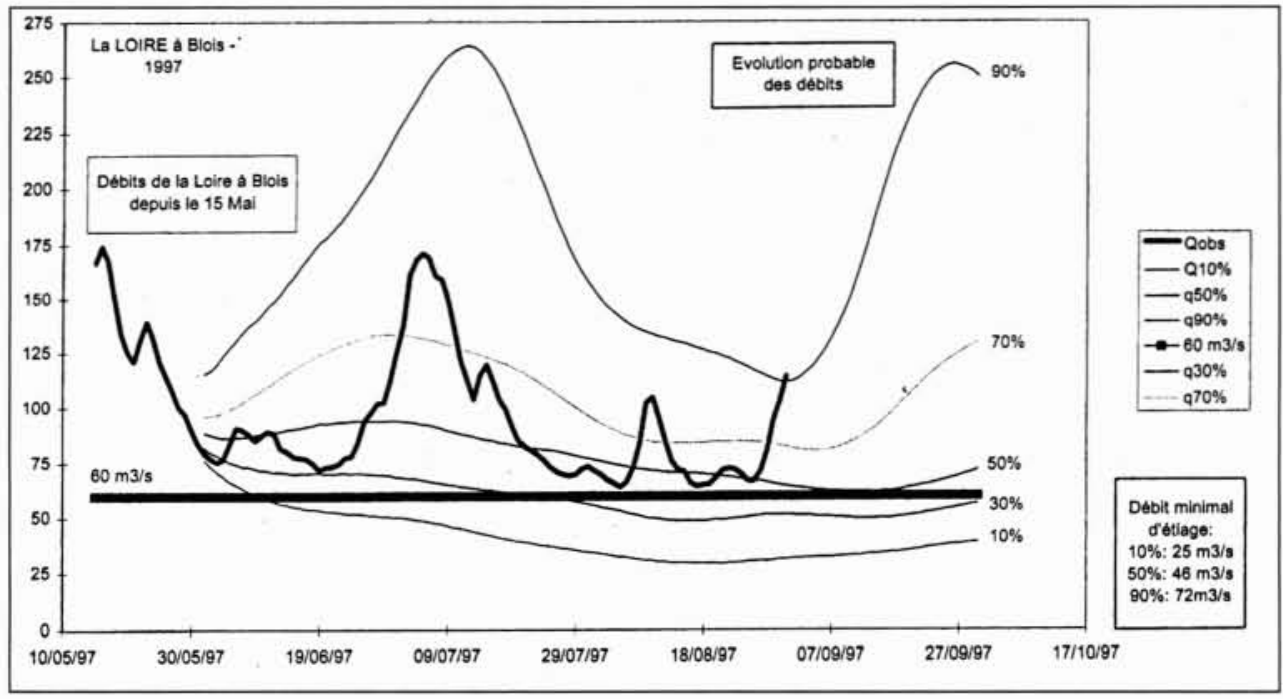

6. Retour d'expérience sur les prévisions statistiques élaborées à partir de la prévision de la courbe enveloppe. compte. Ainsi, pour l'échéance du $1^{\text {er }}$ juin, le faisceau de courbes enveloppes situait le niveau de l'étiage début juillet entre 35 et $60 \mathrm{~m}^{3} / \mathrm{s}$. Le traitement statistique donnait, quant à lui, une chance sur deux au débit de remonter durant le mois de juin, et même $30 \%$ de chance de remonter au-dessus de $130 \mathrm{~m}^{3} / \mathrm{s}$, ce qui s'est effectivement produit suite aux épisodes de précipitations de la fin juin ( voir figure 6).

\section{- 3.4 Vers une aide à la gestion}

La méthode exposée précédemment a permis de répondre rapidement et de façon pratique à une situation extrême. Cependant, elle a été construite dans l'urgence et reste imparfaite tant dans la sélection des années analogues que par le biais introduit par la courbe enveloppe lors d'épisodes pluvieux importants. D'autre part, l'influence du soutien d'étiage n'est pas prise en compte et ce type de résultat ne permet pas d'appréhender d'autre composante que le débit comme la température de l'eau.

Suite à ce constat, une proposition a été faite aux exploitants du bassin de la Loire. Elle consiste à se doter d'un outil d'aide à la gestion. Cet outil permettrait comme sur la Vienne de faire un constat rapide de l'état hydrologique du bassin et de mettre en œuvre des modèles de prévision plus " physiques " tel le modèle MORDOR présenté précédemment, ainsi qu'une modélisation simplifiée de l'évolution de la température de l'eau ou de la gestion des bâches de stockage des effluents. D'autre part, dans le cadre d'un outil d'aide à la décision, suivant la répartition des sous-bassins considérés, une modélisation du soutien d'étiage peut être prise en compte.

Ce projet étant au stade de proposition, on ne le décrit pas plus. Cependant, on présente par la suite le principe de prévision à moyen-long terme des débits naturels.

\subsection{Prévision à moyen terme des débits}

Contrairement à la démarche actuelle qui repose sur un principe statistique, la proposition ci-dessus inclut une modélisation physique des débits et une prévision à moyen terme.

Pour cela, on utilise le modèle MORDOR sur un ou plusieurs sous-bassins suivant l'hétérogénéité des phénomènes climatiques. Ces modèles, appréciés en simulation sur le passé (état hydrologique du bassin), sont soumis à des scénarios historiques sur le futur. Ainsi, on peut appréhender l'évolution possible des débits en considérant le passé hydrologique au jour $\mathrm{j}$ : s'il $\mathrm{y}$ avait dans les mois à venir les conditions météorologiques de l'année $\mathrm{n}$, le débit évoluerait...

La référence à une année donnée peut permettre d'illustrer une situation. La généralisation de ce processus à l'ensemble de l'historique complété par un traitement statistique permet d'aboutir facilement à un ensemble de résultats équivalents à ceux décrits plus haut (nombre de jours sous seuil, débit minimal d'étiage...). Cette démarche permet de s'affranchir des difficultés techniques évoquées précédemment et peut être à l'origine de précision supplémentaire concernant la température de l'eau par exemple. 